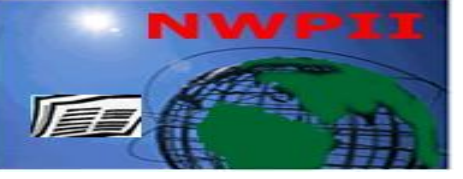

American Journal of Biomedical Sciences

ISSN: 1937-9080

nwpii.com/ajbms

\title{
Physiological Assessment of Driving Mental Fatigue Using Wavelet Packet Energy and Random Forests
}

\author{
Chunlin Zhao', ${ }^{1,}$, Chongxun Zheng', Min Zhao', Jianping Liu ${ }^{2}$ \\ ${ }^{1}$ Key laboratory of Biomedical Information Engineering of Education Ministry, Xi'an Jiaotong University \\ ${ }^{2}$ Engineering College of Armed Police Force. Xi'an 710049, China) \\ *Corresponding Author: \\ Chongxun Zheng \\ Institute of Biomedical Engineering of Xi' an Jiaotong University \\ No.28. Xianning West Road. Xi'an, Shanxi, 710049. P.R. China \\ phone: 86-029-82669055 \\ Email: cxzheng@main.xjtu.edu.cn
}

\begin{abstract}
:
This study measured multiple physiological parameters of mental fatigue induced by the simulated driving task. The subjects' mannerisms and driving performance reveal that the driving mental fatigue is induced after long-term simulated driving. The wavelet packet energy (WPE) of EEG were used to extract the features of brain activity in the normal and the mental fatigue states, then random forests (RF) was employed to select key features for differentiating the two states. The experimental results show that the WPE of EEG and the amplitude of P300 are strongly related with driving mental fatigue. The power spectral indices of heart rate variability (HRV) indicate that the sympathetic nervous system may play a leading role and the parasympathetic nervous system is retrained at the end of driving task. Furthermore, RF method can select features effectively, reduce the dimensionality of the feature vectors significantly, and then achieve a recognition accuracy of $97 \%$. The WPE-RF method could be a potential way of assessment of driving mental fatigue.
\end{abstract}

Key words: EEG; WPE; P300; HRV; RF; driving mental fatigue

Received: 27 December 2009; | Revised: 7 March 2010; | Accepted: 12 April 2010

\section{Introduction}

Mental fatigue is a common phenomenon in our daily life, and is defined as a state of cortical deactivation, which reduces mental performance and decreases alertness [1]. The major symptom of mental fatigue is a general sensation of weariness, feelings of inhibition, and impaired activity. Especially in transportation and aviation area, mental fatigue is a main cause of some serious operating errors. Driver's mental fatigue was believed to account for $20-30 \%$ of all traffic accidents according to the report by the Parliament of the Commonwealth of Australia [2]. Fatigue related crashes are often more severe than others because the driver's reaction times increased, or they have failed to make any maneuvers to avoid a crash. Thus, developing and establishing a more 
reliable and accurate method for evaluating mental fatigue is necessary to reduce operating risk, improve productive efficiency and maintain people's health.

From the literatures, we can see two categories of efforts on the measurement mental fatigue. One aims to measure the vehicle status, such as steering angle, vehicle lateral position, vehicle speed etc [3-4]. However, these parameters might vary with the vehicle type, driver's experience, geometric characteristics and state of the road, thus the measurements might require devising different detection logics. The other focuses on measuring indicators related to the human body which include self-report, performance, physical behavior, perceptual, electrophysiological, psychological and biochemical measurements etc [5]. In the last decade, many researchers began to study the development of the monitoring systems using accurate detection techniques that were mostly based on physiological phenomenon like EEG, ECG, heart rate, pulse rate and respiration. There were several EEG studies related to mental fatigue in the past. Some studies reported EEG spectral changes as alertness declines. For example, the proportion of low-frequency EEG waves, such as theta and alpha rhythms, might increase while higher-frequency waves, such as beta rhythms might decrease [6-7]. Schier (2000) used a driving simulator to observe EEG during the driving task. The results showed that the relative power of alpha increased when the attention level of the driver decreased [8]. Lal and Craig (2003) found significant changes in theta, delta, alpha and beta of EEG during driving mental fatigue [5]. Lin C.T et al (2007) demonstrated the feasibility of detecting and analyzing multiple streams of ERP signals that represent operators' cognitive states and response to driving task events [9]. A few studies even showed that the average heart rate was of slightly increasing frequency after driving experiments [10]. While numerous physiological indicators are available to measure mental fatigue, the EEG signal may be one of the most predictive and reliable [1]. Some scholars reported that performing monotonous tasks was related to the increase of the $0.1 \mathrm{~Hz}$ component in the HRV [11]. Other studies explored the links between fatigue and changes in event-related potential (ERP) components. Mental fatigue was found to produce a decrease in P300 amplitude while latency increases [12].

However, driving mental fatigue is a complex phenomenon involving physical, psychosocial, and behavioral processes, and no single-parameter measures could be sufficiently sensitive and reliable enough to quantify driving mental fatigue. So a multiple-measure approach is needed to develop a robust method of fatigue detection

In this paper, we incorporate multiple measures to assess driving mental fatigue. The subjective self-reporting measures and the react time (RT) to visual oddball task stimuli were utilized to verify that long term driving task would induce mental fatigue to the subjects. Then the blink frequency, the P300 amplitude and latency based on visual oddball task, the power spectral parameters of HRV and multichannel wavelet packet parameters in four frequency bands of EEG were applied to estimate driving mental fatigue. Finally, random forest was used to identify the key features of driving mental fatigue and differentiate pre- and post-task mental fatigue states based on multichannel wavelet packet parameters of EEG.

\section{Methodology}

\subsection{Subjects}

To reduce inter-subject and age differences, thirteen male graduate students (mean age, 23.2 years; range 22-26 years) were recruited from students of Xi'an JiaoTong University to perform the experiments. They all have not obtained any driving experience before the simulated driving. None of them worked night shifts, used prescription medication, or having any medical contraindications, psychological or intellectual problems. All subjects have normal or correctedto-normal vision and were right-hand dominated. All subjects were trained about one week before simulated driving task until they could perform the simulated system expertly. Subjects were required to sleep adequately one day before the test and refrain from smoking, or consuming alcohol, caffeine, tea or food approximately $12 \mathrm{~h}$ before the study. 


\subsection{Apparatus}

The driver simulator equipment consisted of a car frame with built-in steering wheel, gas and brake pedals, clutch, manual shift and a horn and turn signal, etc. The visual display of the VRbased driving simulated environment is a $19 \mathrm{~cm}$ Liquid Crystal Display (LCD) at a distance of 80 $\mathrm{cm}$ from the subject's eyes. The LCD shows other cars, the current speed and other road stimuli. The system also provided engine noise and nearby traffic noise. The simulated route and traffic sign were standardized with national traffic law.

\subsection{Experiment Design}

Previous literatures reported that the driving mental fatigue usually occurred in a monotonous driving environment [1]. Taking this factor into account, the highway scene was selected in this study. There were few scenery changes and moving targets in three-lane road, in order to reduce the outside stimuli for the subjects. A very light curvature was chosen so that the subjects could focus on steering all the time (see fig.1).

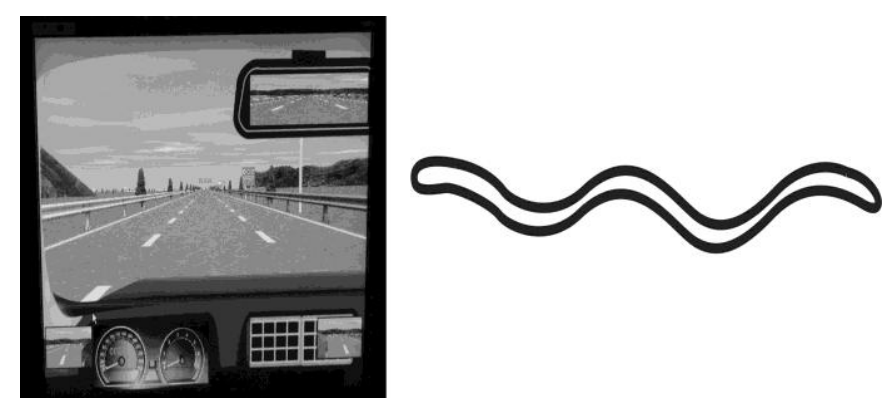

Figure 1. The simulated scene and route

This study was conducted in a soundattenuated and temperature-controlled laboratory. Experimental procedure was as follows. Training was performed in advance. The experiments began at 9:00 AM or 3:00 PM and lasted for 2.5 hours. The time was consistent with the work-time on normal workdays. Before the experiment, the subjects were instructed to well understand the procedure and the instructions. At the beginning of driving task, subjects were required to finish the psychological self-report measures of mental fatigue, and then an oddball task was conducted. In this task, a red and a green circular image were randomly displayed on the LCD. Each circular image was presented on screen for $160 \mathrm{~ms}$. The probabilities of appearance of red signal and green signal were 0.85 and 0.15 respectively. The interstimulus intervals varied randomly between 1000 and $1200 \mathrm{~ms}$. The 200ms EEG data before stimulus onset and the $800 \mathrm{~ms}$ EEG data after stimulus onset were used for averaging ERPs. Subjects should respond to target stimuli as quickly as possible. During the driving task, subjects operated the simulator for 90 minutes without any break. Subjects were asked to restrain from unnecessary movements as much as possible, so that they can maintain constant speed and avoid car crash. There were not any questionnaires. At the end of all experiment sessions, another oddball task (the different stimulus sequence) and the same psychological self-report measure of fatigue were also carried out.

\subsection{Data Acquisition}

The Neuroscan Synamps system (Synamps ${ }^{2}$, Scan 4.3, Ei Paso, Tex, USA) was used to record the physiological data. Thirty channels of EEG, one channel of ECG, horizontal and vertical EOG were measured simultaneously while participants were performing experimental tasks. The $10-20$ international standard of electrode placement was applied. The impedance was kept below 5k for EEG region and 10k for EOG and ECG region. All physiological signals were sampled at $500 \mathrm{~Hz}$ with $0.05 \mathrm{~Hz}-70 \mathrm{~Hz}$ band-pass filter and $50 \mathrm{~Hz}$ notched.

EEG raw data are always contaminated with noise. Firstly, the data were visually inspected and data segments containing possible residual artifacts were eliminated. EEG over $100 \mathrm{uV}$ was also treated as artifacts. EOG artifacts were removed by using EOG signals as predictors of the artifact voltages at each EEG electrode in a multivariate linear regression. ECG raw data were treated by wavelet-denoising. The measurements were carried out at two epochs: the beginning and the end of driving task.

During the whole simulated driving, the mental fatigue signs such as rubbing, yawning and nodding etc, the driving performances such as car accidents, flameout, and other operating errors were recorded manually by an observer to validate mental fatigue states. 


\subsection{HRV Analysis}

HRV is a useful signal for understanding the status of the ANS. It is a powerful means of observing the interplay between the sympathetic and parasympathetic nervous systems. The balancing action of the sympathetic nervous system (SNS) and parasympathetic nervous system (PNS) branches of the ANS controls the HR. Increased SNS or diminished PNS activity results in cardio-acceleration. Conversely, a low SNS activity or a high PNS activity causes cardiodeceleration. High-frequency (HF:0.15-0.40 Hz) spectral power is used as an index of parasympathetic activity and low-frequency (LF: $0.04-0.15 \mathrm{~Hz}$ ) spectral power as that of sympathetic and parasympathetic activity. LF/HF ratio is used to indicate balance between sympathetic and parasympathetic tone [13-14].

\subsection{ERP Analysis}

ERP is any measured brain response that is directly the result of a thought or perception. To see the brain response to the stimulus, the experimenter must conduct many trials (100 or more) and average the results together, causing random brain activity to be averaged out and the relevant ERP to remain. The event-related brain potential (ERP) is a transient series of voltage oscillations in the brain that can be recorded on the scalp following a discrete stimulus event [15]. ERP is strongly related to the level of attention. The prolonged P300 latency that indicated the delayed cognitive information processing and the decreased P300 amplitude that is related to the decreased activity of cognitive information processing were found to be effective measures of mental fatigue [16]. At the beginning and end of driving task, the subjects were asked to finish an oddball task respectively. The 200ms EEG data before stimulus onset and the 800ms EEG data after stimulus onset were used for averaging EPR. The clear P300 waveform could be obtained after averaged off-line.

\subsection{Wavelet Packet Analysis for EEG}

Wavelet packet analysis can provide more subtle information about signals not only in approximation space but also the detail space.
Both approximation space and detail space were decomposed into new lower resolution approximation spaces and detail spaces simultaneously [17-18]. $\phi(t)$ and $\psi(t)$ were defined as the scaling function and the corresponding mother wavelet function respectively. Supposing $\psi^{0}(t)=\phi(t), \psi^{1}(t)=\psi(t)$, can construct the wavelet basis as follows according to two-scale equations.

$$
\begin{aligned}
& \psi_{j, k}^{2 i}(t)=\frac{1}{\sqrt{2}} \psi^{2 i}\left(\frac{2^{j} k-t}{2^{j}}\right)=\sum_{n} h(n) \psi_{j-1,2 k-n}^{i}(t) \\
& \psi_{j, k}^{2 i+1}(t)=\frac{1}{\sqrt{2}} \psi^{2 i+1}\left(\frac{2^{j} k-t}{2^{j}}\right)=\sum_{n} g(n) \psi_{j-1,2 k-n}^{i}(t)
\end{aligned}
$$

where $i$ is the node number, $j$ is the level of decomposition. $h(n)$ and $g(n)=(-1)^{1-n} h(1-n)$ are a pair of quadrature mirror filters group. Base on the equation (1) and (2), the decomposed wavelet packet coefficients with different $j$ th level and $k$ th point of signal $f(t)$ can be calculated by recursion formula as follows:

$$
\begin{aligned}
& d_{j}^{2 i}(k)=\int f(t) \psi_{j, k}^{2 i}(t) d t=\sum_{n} h(n) d_{j-1}^{i}(2 k-n) \\
& d_{j}^{2 i+1}(k)=\int f(t) \psi_{j, k}^{2 i+1}(t) d t=\sum_{n} g(n) d_{j-1}^{i}(2 k-n)
\end{aligned}
$$

The sub-signal of the sub-band space on the $j$ th level, can be reconstructed by:

$$
s(t)_{j}^{2 i}=\sum_{k} d_{j}^{2 i}(k) \psi_{j, k}^{2 i}(t)
$$

Since the wavelet $\psi_{j, k}^{2 i}(t)$ is an orthogonal basis at $L^{2}(R)$, the $j$ th level scale-energy of the sub signal $s(t)_{j}^{2 i}$ is calculated by:

$$
E_{j}=\sum_{k}\left|d_{j}^{2 i}(k)\right|^{2}
$$

In consequence, the total energy can be obtained by:

$$
E_{\text {total }}=\sum_{j} E_{j}
$$

Then the relative WPE for the resolution level $j$, denoted by $p_{j}$, is defined as 


$$
p_{j}=\frac{E_{j}}{E_{\text {total }}}
$$

which quantifies the probability distribution of the spectral energy [19-20].

In this paper, multi-dimension features were extracted from EEG after eight-octave wavelet packet decomposition, which consisted of relative WPE in delta $(0.5-3.5 \mathrm{~Hz})$, theta $(4-7 \mathrm{~Hz})$, alpha $(8-12 \mathrm{~Hz})$, beta $(13-30 \mathrm{~Hz})$ frequency bands and the ratio indices beta/alpha, theta/alpha, (alpha+theta)/beta. These indices of EEG are reported to represent driver mental fatigue [21]. EEG data from ten electrodes (fp1, fp2, f3, f4, c3, $\mathrm{c} 4, \mathrm{p} 3, \mathrm{p} 4, \mathrm{o} 1, \mathrm{o} 2)$ will be presented as being representative of prefrontal, frontal, central, parietal and occipital regions. Above mentioned indices of all EEG data segments were calculated for every electrode respectively, the features of 70 dimensions for every EEG data segment were obtained.

\subsection{Random Forest}

Recently, combined classifiers, which show better performance than the single classifier does and can avoid over-fitting, are widely used in machine learning field [22]. Random Forest (RF) developed by Leo Breiman is a multi-classifiers fusion algorithm that gives the most accurate prediction in classification [23].

$\mathrm{RF}$ is an ensemble of many randomized decision-trees $\left\{h\left(x, \Theta_{k}\right), k=1,2, \ldots, n\right\}$, in which, $\Theta_{k}$ is a group of independent indentical distribution random vectors. Decision-tree is the smallest unit of RF. Each decision-tree depends on both the bootstrap training set and the random input selection for determining the split at each node during the tree growing. The testing case is fed down through each decision tree in the forest, for each tree makes its local decision. Then the final decision is determined through a majority vote.

A forest is grown by using ntree bootstrapped samples of size $n$ randomly drawn from the original data of $n$ points with replacement. This first type of randomization helps build an ensemble of trees and reduce dependence among the trees. About two-thirds of the data set is used to grow a classification tree. About one-third of the data are reserved as Out of Bag (OOB) Data. The second type of randomness is used during building classification trees. For each node of a tree, the RF algorithm randomly selects $m_{t r y}$ variables and uses only them to determine the best possible split using the Gini index as the splitting criterion. This algorithm is fairly robust to the choice of the number $m_{t r y}$, the value of which is usually taken to be the square root of the total number of variables.

When applying the random forest algorithm, the OOB data is used. Each tree in the forest is grown using the bootstrapped sample, and the OOB part is subsequently processed by the grown tree. This gives rise to classification for each point in the OOB part of that bootstrapped sample, which means that about one-third of the trees in the random forest give a prediction for each point in the original data. The final classification of a particular data point is decided on the basis of majority vote or user defined threshold, among the trees predicting that data point. The unbiased estimates of true classification rates are calculated by comparing the OOB set classification made by the forest to the experimentally observed classes to which the data points belong.

Another valuable property of RF is that it can give an importance estimate for all the features. After the whole RF is constructed, the raw importance score for $m^{\text {th }}$ feature is defined as:

$$
\sum_{i}\left(\text { Count }_{i}-\text { Count }_{i}{ }^{m-\text { permuted }}\right) / N
$$

where $N$ is the total number of trees in RF, and Count ${ }_{i}$ or Count ${ }_{i}{ }^{m-p e r m u t e d}$ is the count of the number of votes cast for the correct class for all the OOB case or variable-m-randomly-permuted OOB cases of $i^{\text {th }}$ tree respectively [23].

\section{Results}

\subsection{Self-report}

Subjects all felt tired, bored and drowsy when the driving task was over. They were yawning more or less during driving task and reported 
difficulties in concentrating and focusing their attention on the driving task. They also reported that these feelings became stronger and there were difficulties to concentrate and focus their attention on the driving task with driving time increasing. These evidences indicated that driving mental fatigue was induced after 90 min driving task. According to Li's subjective fatigue scale (LSFS) [24] (the subjective scores ranged from 1 to 7 , a scale which represented no, minimally, somewhat, a little, considerably, very and extremely), the mental fatigue scores increased from 1.31 to 5.46 $(\mathrm{P}<0.005)$ at the end of task.

\subsection{Some Objective Indicators of Driving Mental Fatigue}

To maintain scientific validity, questionnaires should not be the sole identifier of driving mental fatigue symptoms. More objective measures need to be combined to assess the state of driving mental fatigue. The subject's mannerisms such as rubbing, yawning and nodding, the driving performance such as car crashes, flameout, and other operating errors, the react time (RT) and error ratio in oddball task and the vertical EOG were combined to validate the different driving mental fatigue status. The EOG was used to identify blink artifact in the EEG data as well as changes in blink types such as the small and slow blinks that characterize fatigues.

Table 1 shows the proportion of subjects categorized according to the mannerisms identified from the manually recorded data as well as the driving performance in two epochs. There were almost no fatigue physical mannerisms at the beginning of the driving task. But these mannerisms were observed from all subjects at the end of the driving task. The lapses of driving performance also increased linearly with driving time increasing. Over $70 \%$ subjects in the operation made errors during fatigue state.

Table 1. The mannerisms of fatigue and lapses in driving performance

\begin{tabular}{lcc}
\hline Mannerisms & the Beginning & the End \\
\hline Rubbing & $6.67 \%$ & $100 \%$ \\
Yawns & $0 \%$ & $100 \%$ \\
Noddings & $0 \%$ & $100 \%$ \\
\hline
\end{tabular}

\begin{tabular}{lcc}
\hline Flameout & $0 \%$ & $73.33 \%$ \\
Car accidents(collisions) & $0 \%$ & $93.33 \%$ \\
Other driving errors & $0 \%$ & $80 \%$ \\
\hline
\end{tabular}

(In table1, each validated mannerisms for subjects should satisfy these criterions respectively: 1 Rubbing/5 min; 1 Yawn/5 min; 1 Noddings/30s; 1 Flameout/7.5 min; 1 Car accidents/7.5 min; 1 Other driving errors/15 min.)

The oddball task required subjects to respond to target stimuli as quickly as possible. The paired t-test analysis shows that there was statistical difference between two epochs in RT ( $\mathrm{t}=-3.946$, $\mathrm{p}=0.002)$, the average RT increased from $356.35 \mathrm{~ms}$ to $376.65 \mathrm{~ms}$ at the end of the driving task.

The blink frequency can be estimated by EOG. The data of blinks were obtained from EOG by identifying the peak of blink based on wavelet detection method. The results showed a highly statistical difference between the two epochs. ( $\mathrm{t}=-$ $3.224, \mathrm{p}=0.007$ ), and the mean value of blink frequency increased from 14.7 to 20.7 beat/min at the end of driving task.

\subsection{Power Spectral of HRV}

The 5min of ECG at the beginning and end of the driving task was selected for analysis. The detection of $\mathrm{R}$ waves is done with an algorithm based on wavelet transforms. The HRV signal was obtained from the sequence of RR interval. The power spectrum of HRV was estimated using AR model (order 20). Fig.2 shows the HRV result at two epochs respectively. Comparing two epochs, the LF was statistical different $(\mathrm{t}=-3.1, \mathrm{p}=0.009)$, the power increased from a mean of 732.7 $(\mathrm{SD}=434.44)$ to 1057.5 ( $\mathrm{SD}=637.42)$; At the same time, the high frequency component was also statistically different $(\mathrm{t}=2.32, \mathrm{p}=0.039)$, in contrast to the LF, the power of HF decreased from a mean of $859.03(\mathrm{SD}=77.4)$ to $626.18(\mathrm{SD}=54.1)$. The ratio $\mathrm{LF} / \mathrm{HF}$ increased significantly from a mean of $1.42(\mathrm{SD} 0.27)$ to $2.34 \quad(\mathrm{SD} 0.29)(\mathrm{t}=-4.24$, $\mathrm{p}=0.001)$. 


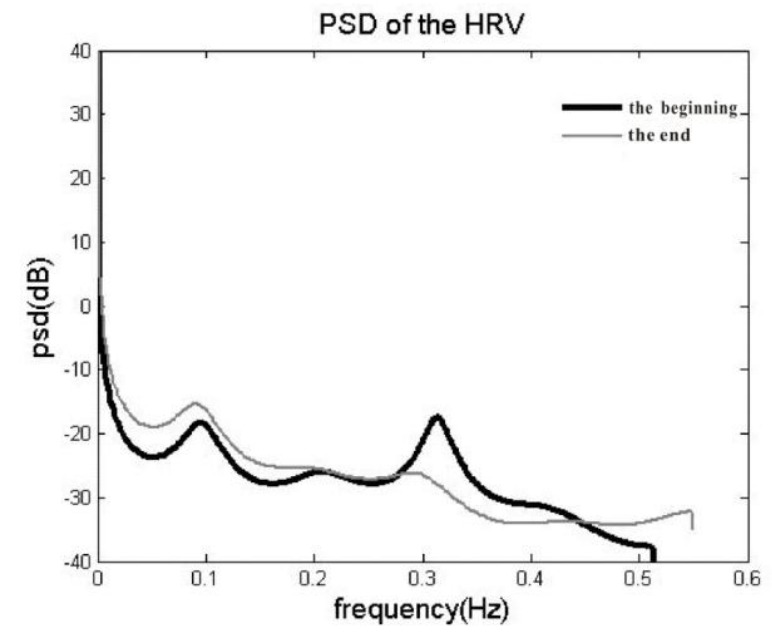

Figure 2. The PSD of HRV between two task epochs

\subsection{ERP Analysis}

The P300 amplitude at Fz, Cz, Pz electrodes were calculated using the base-peak method which searched within a window from 300 to $800 \mathrm{~ms}$ for the maximally positive segment average of $100 \mathrm{~ms}$ and subtracted the pre-stimuli $100 \mathrm{~ms}$ baseline to obtain base-peak measures. The midpoint of the maximum positive segment was defined as P300 latency. Fig.3 shows the P300 waveform in tow epochs. (the dark line represents P300 at the beginning of task, the dark-grey line represents P300 at the end of task).

\subsection{Wavelet Packet Parameters of EEG}

After artifact detection and ocular correction, 30 seconds continuous EEG data for each subject in the session of pre-task and post-task were analyzed. The first 10s EEG data was chosen as basic data segment and 0.2 second as step length. By shifting the basic data segment with step length, 2600 data segments were obtained totally.
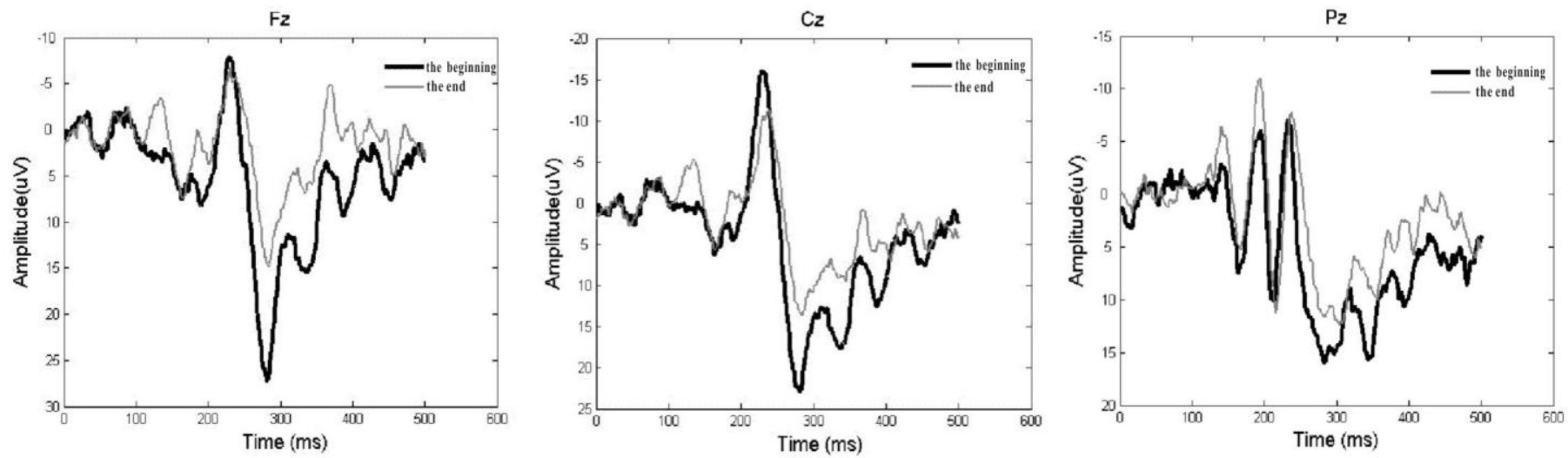

Figure 3. ERP waveforms on $\mathrm{Fz}, \mathrm{Cz}$ and $\mathrm{Pz}$ electrodes between two task sessions.

The latency of P300 did not show statistical difference with task time. To amplitude of P300, except $\mathrm{Pz}$ location, there were significant difference between pre-driving and post-driving $(\mathrm{t}(\mathrm{Fz})=4.791$, $\mathrm{p}<0.001 ; \mathrm{t}(\mathrm{Cz})=2.527, \mathrm{p}=0.027 ; \mathrm{t}(\mathrm{Pz})=0.273, \mathrm{p}=0.790)$. The amplitude of $\mathrm{P} 300$ decreased at the end of driving task.

When applying wavelet packet analysis to every EEG data segment, relative WPE indices in four frequency bands and their ratio indices beta/alpha, theta/alpha, (alpha+theta)/beta on 10 electrodes were calculated. To eliminate the influences of parameter's fluctuation, the WPE mean values of 10 electrodes ( $\mathrm{fp} 1, \mathrm{fp} 2, \mathrm{f} 3, \mathrm{f} 4, \mathrm{c} 3$, c4, p3, p4, o1, o2) EEG was calculated to be statistically analyzed. Compared to two epochs, there were no statistical differences in relative WPE of delta and ratio index beta/alpha for all electrodes location. But the mean values of relative WPE in theta and alpha bands significantly increased $(\mathrm{P}<0.05)$, while that in beta bands significantly decreased $(\mathrm{P}<0.05)$ on prefrontal, frontal, central and occipital electrodes. The mean values of theta/alpha increased and that of (alpha+theta)/beta increased significantly $(\mathrm{P}<0.05)$ on prefrontal, frontal and central electrodes. The results of wavelet packet 

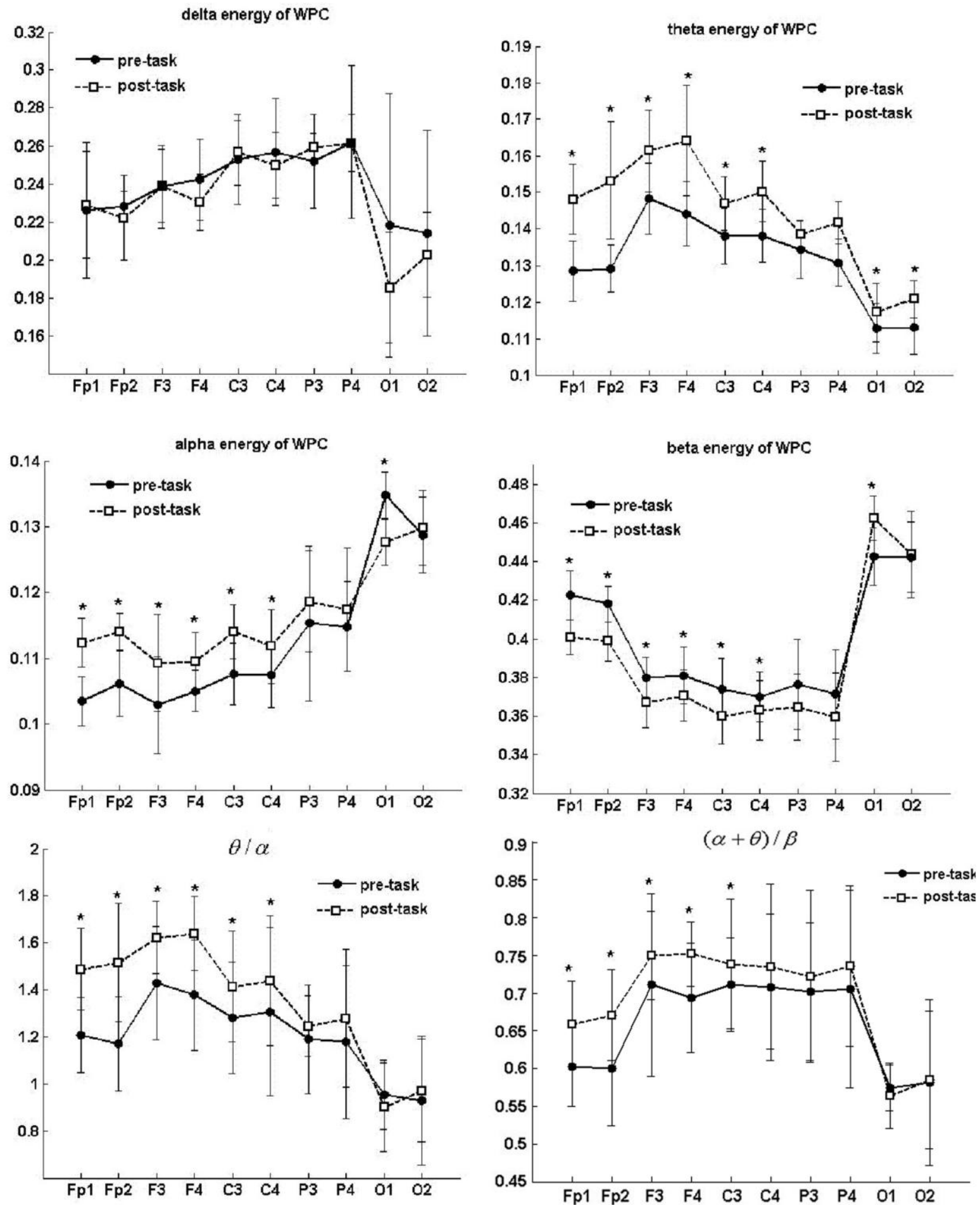

Figure 4. Wavelet packet parameter of EEG at two sessions. (* $\left.{ }^{*} p<0.05\right)$

The results indicated the wavelet packet parameters of EEG changed between two epochs and are closely related with driving mental fatigue.

\subsection{Key Feature Indentify and Classification Based on RF}

$\mathrm{RF}$ can estimate the importance of variables by OOB data. Fig.5 shows the result of all features. From Fig.5, it can be seen that not every 
features has the same significance for the identification of driving mental fatigue. The major contributive features are fixed on theta, alpha, beta bands, ratio 2 and ratio3.

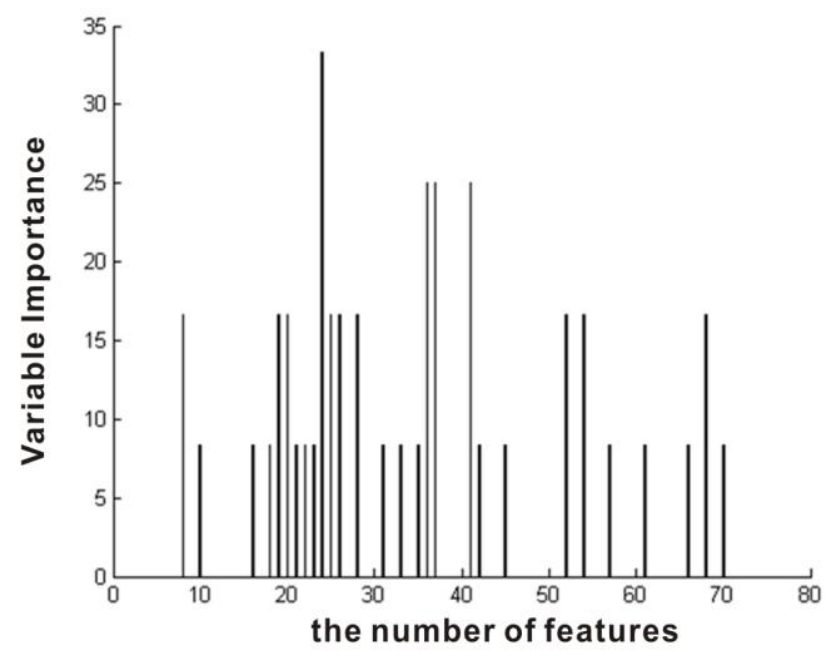

Figure 5. The importance estimation for all features (the number of features represents the different bands of EEG and relative ratio on $\mathrm{fp} 1, \mathrm{fp} 2, \mathrm{f} 3, \mathrm{f} 4$, c3, c4, p3, p4, o1, o2 electrode position. 1-10: delta, 11-20: theta, 21-30: alpha, 31-40: beta, 4150: beta/alpha, 51-60: theta/alpha, 61-70: (alpha+theta)/beta)

Fig.6 shows the relationship between classification errors based on OOB data and the numbers of trees during training session. When the number of trees is more than 100, the errors are below $1 \%$. In growing a forest, the number of trees is an important parameter, to ensure the convergence of forest and smaller errors. In this study, in order to reduce training time span and reach higher classification accuracy, the numbers of tree were determined as 100.

As the number of EEG data segment available was limited, a 5-fold cross-validation test was applied. The data sample set was randomly averaged into five portions, each trail uses one portion as testing data and the other four as training data, and different trials used different testing sets. The classification accuracy was calculated over 5 trials with different random selection of training and testing set. Table 2 shows the classification accuracy for RF and SVM.

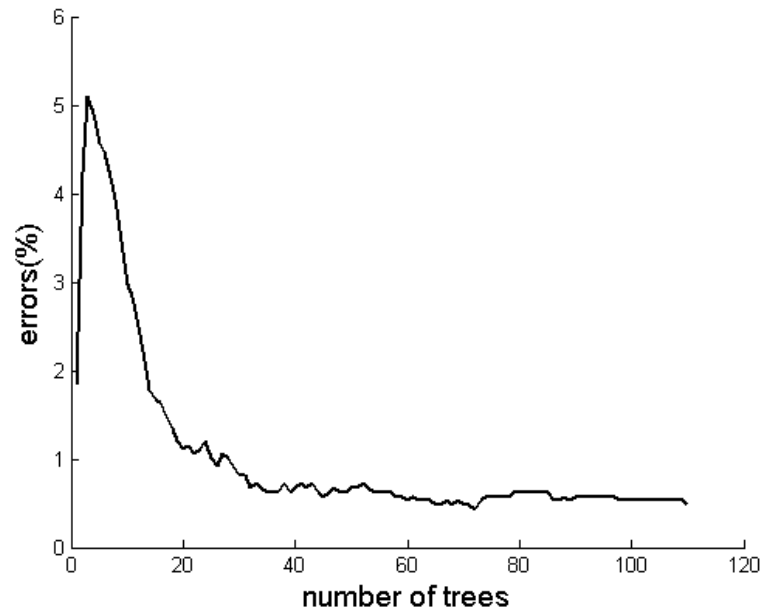

Figure 6. The relationship between errors and numbers of trees

Table 2. The classification accuracy

\begin{tabular}{ccc}
\hline & RF & SVM \\
\hline Pre-task & $98 \%$ & $99 \%$ \\
\hline Post-task & $96 \%$ & $96 \%$ \\
\hline
\end{tabular}

RF and Support Vector Machines (SVM) are two algorithms that currently give the most accurate prediction in classification. Table 2 compares the result based on the two algorithms. It can be concluded that the performance of RF and SVM is at the same level.

\section{Discussion}

Driving mental fatigue is a complicated phenomenon that deals with physiology and psychology. There is the lack of so-called "gold standard" definitions to describe such phenomenon. There are various factors that induce driving mental fatigue, such as long-time driving, the monotonous operation environment, etc. Especially, long periods of monotony negatively impact driver alertness, vigilance and driving performance $[3,10]$. This study focused on the driver mental fatigue status under monotonous and repetitious highway scene in simulated laboratory condition.

Craig et al (2006) investigated the psychological determinations of mental fatigue. 
They found that the self-report score contributed strongly to psychological outcome measures [25]. With time on task passing, the subjective fatigue score increased significantly and some physical signs of mental fatigue such as rubbing, yawning and nodding, etc. occurred on every subject after long term driving. It is well known that driving mental fatigue can influence performance and cognitive response, which is thought to be associated with reduced efficiency and alertness, prolongation of cognitive information processing, difficulty in concentrating notice, impaired mental performance etc [26-28]. Maybe this is a reasonable explanation for the progressive increase in driving errors. The self-report measures were also linked to EEG WPE indicators of fatigue such as theta, alpha, and beta variability as well as the EOG changes. In our study, the conventional blinks during the alert phase are replaced by fast rhythmic blinks during mental fatigue. The blink frequency increases positively with the extent of mental fatigue. This result is consistent with what S.K.L Lal (2002) reported. The conventional blinks during the alert phase were replaced by fast rhythmic blinks during mental fatigue.

Previous studies have reported that heart rate was the most sensitive cardiovascular index of the workload and fatigue associated with driving vehicle [29]. Some literatures reported heart rate change during certain driving tasks [30]. Heart rate is primarily controlled by the autonomic nervous system (ANS), and may be increased by shifting sympathovagal balance caused by driving mental fatigue. Sympathetic activity is associated with the LF while parasympathetic activity is associated with the HF of modulation frequencies of the heart rate. The $\mathrm{LF} / \mathrm{HF}$ ratio is often used as a metric of sympathetic-parasympathetic balance [31-33]. In general condition, the sympathetic nervous system usually works in a tense status. The parasympathetic nervous system works in a mental peace condition, thus the physiological response will be opposite to the sympathetic nervous system. As already mentioned in the introduction, there is a strong link between mental fatigue and the autonomic nervous activity, and the performing of monotonous tasks is related to the increase of the LF component in HRV. In this study, the power of $\mathrm{LF}$ and $\mathrm{LF} / \mathrm{HF}$ ratio is significantly higher and the power of $\mathrm{HF}$ significantly lower after 90min driving task than that of the pre-task period. Therefore it is evident that the sympathetic nervous system plays a leading role and the parasympathetic nervous system retrained in the fatigue state. The predominant activity of autonomic nervous system of subjects turns to the sympathetic activity from parasympathetic activity after the driving task.

It is well known that the amplitude and latency of P300 in ERPs evoked by performing the oddball paradigm reflect the extent of individuals' attention and cognitive information processing which were found to be effective measures of mental fatigue [34]. Uetake and Murata (2005) pointed out that the event-related potential (ERP) components (such as P300) were useful to identify the depth of cognitive information processing. They found that the prolonged latency indicates the delayed cognitive information processing and the decreased P300 amplitude was related to the decreased activity of cognitive information processing [16]. We only find the amplitude decreases significantly at the end of driving task. The p300 amplitude reflects the depth or degree of cognitively processing the stimulus. In other words, it is strongly related to the level of attention. The decreased P300 amplitude proves the declination of the level of attention after long time driving, which could be a significant cause of road accidents. The increase in RT to stimulus after driving task should be associated with driving mental fatigue, which is evident by subjective self-report measures. Some researchers proposed an Asynchrony Model in which the extent of neural asynchrony is proportional to the conditioned foot reaction time to visual events while in a driving-like scenario by MEG and fMRI studies [35-36]. Whether the longer RT to oddball task in our study can be explained by the Asynchrony Model still demands a further verifying study.

It has been known for many years that changes in brain arousal involve specific changes in oscillatory brain activity. Wavelet packet transform can generate spectral resolution fine enough to meet the problem requirement. We investigate the wavelet packet energy features of 
EEG from prefrontal, frontal, central, parietal and occipital electrodes. Except parietal region, most of the relative WPE and ratio parameters on the other regions show significant difference between the beginning and the end of driving task. Smith and Givens have demonstrated regional differences in the manner in which particular task demands modulate the EEG spectrum [37]. Some brain regions are of particular importance in maintaining the primary driving performance of event detection during a secondary conversation task. Several researchers have confirmed this conclusion by using both fMRI and MEG methods [35-36]. Maybe it is a good explanation for the EEG rhythm changes on different scalp regions.

At the same time, relative WPE of alpha and theta rhythms increase significantly and relative WPE of beta rhythm decreases significantly $(\mathrm{P}<0.05)$. Based on previous reports, the beta rhythm is associated with increased alertness and arousal, and the decrease of beta energy implies that the brain arousal level declines. The alpha rhythm occurs during relaxed conditions, at decreased attention levels and in a drowsy but wakeful state. The magnitude of alpha activity during cognitive tasks was inversely proportional to the number of cortical neurons recruited into a transient functional network for task performance. The increase of alpha energy indicates that the number of activating cortical neurons is decreasing with driving duration, and the cortical activity is inhibiting. The theta rhythm mainly occurred at sleep state, and the increase of theta energy is the sign of onset of slight sleepy [38-38]. The energy tendency of alpah, beta, and theta rhythm is consistent with driving mental fatigue status. Three ratio indices also reflect the fluctuation of alpah, beta, theta rhythms and the change of driving mental fatigue. It is suggested that the alertness ability of subject decreases at the end of driving task which induces slow waves of EEG increase and the fast waves decrease. Decision tree is known for its ability to select important descriptors among many features.

Random forest, as an ensemble of trees, inherits this ability and can measure how each descriptor contribute to the prediction accuracy in the course of training. From our result, we can see that alpha, beta, theta rhythm of EEG and their ratio have main contribution to the prediction. Especially, alpha rhythm on each electrode also has contribution. It is similar with the WPE statistical analysis result. During training course, the errors are below $1 \%$ when the numbers of tree is chosen as 100. Nevertheless, there is no increase in computational complexity based on the following reasons: random forest only tests $m_{t r y}$ of the descriptors. And since $m_{t r y}$ is typically very small, the search is very fast; random forest does not do any pruning at all via cross-validation, and it can take up a significant portion of the computations [40]. Compared with SVM, RF takes less computational time without compromise in classification accuracy. So RF has a great application potential in practice.

\section{Conclusion}

Driving mental fatigue is a complicated physiological and psychological process, which induces the changes of psychology, behavior and physiology of subjects. We focus on the physiological approach to estimate driving mental fatigue in simulated laboratory environment. The relative WPE of different EEG rhythm, the amplitude of P300, and power spectrum of HRV have shown significant difference between two epochs. There is a close relationship between driving mental fatigue and the physiological parameters. The subject's mannerisms and driving performance can reflect driving mental fatigue status objectively. Then the RF leads to better classification and faster calculation. The experimental results show that RF algorithm enhances the generalization ability of the classifier and improves the accuracy of driving mental fatigue states recognition.

However there are several limitations to the study. First, we had a relatively small number of participants in the experiment. In order to generalize our results, studies involving a large number of subjects are essential. Second, the subjects used in this study were all males and without any driving experience, which can be short in representing general driver population. Third, since no controls were put in for subjects who have done some other task totally unrelated to 
driving, the physiological measure of mental fatigue may not be specific to driving task. These study limitations will be considered in the future study. In the next phase of our research, we plan to test the feasibility of psychophysiological measurements in both the laboratory and field contexts. However, the psychophysiological measures and WPE-RF model may provide useful insight for future development of a countermeasure and management for driving mental fatigue.

\section{Acknowledgments}

The project is supported by National Science Foundation of China under grant No. 30670534.

\section{References}

[1] Lal, S.K.L.; Craig, A. A critical review of the psychophysiology of driver fatigue, Biol Psychol, 2001, 55, 173-194.

[2] The Parliament of the Commonwealth of Australia. Beyond the midnight oil: Managing fatigue in transport, House of Representatives Standing Committee on Communication, Transport and the Arts, Australia, 2000.

[3] Roman, B,; Hana, K.; Pousek, L.; Smrka, P.; Schreib, P.; Vysoky, P. Detecting of fatigue states of a car driver, ISMDA 2000, Lect Notes Comput Sci, 2000, 1933, 260-273.

[4] Sayed, R.; Eskandarian, A. Unobtrusive drowsiness detection by neural network learning of driver steering, Proceeding of the Institution of Mechanical Engineers, Part D: Journal of Automobile Engineering, 2001, 215(9), 969-975.

[5] Lal, S.K.L.; Craig, A.; Boord, P.; Kirkup, L.; Nguyen, H. Development of an algorithm for an EEG-based dirver fatigue counterment , J Saf Res, 2003, 34(3), 321-328.

[6] Scerbo, M.; Freeman, F.G.; Mikulka, P.J. A bioicybernetic system for adaptive automation, In: Backs,R.W; Boucsein, W. Eds. Engineering Psychophysiology: Issue and Applications, Mahwah: Lwarence Erbbaum Associated, 2000, 241-253.

[7] Klimesch, W. EEG alpha and thea oscillations reflect cognitive and memory performance: a review and analysis, Brain Res Rev, 1999, 29(2-3), 163-195.

[8] Schier, M.A. Changes in EEG alpha power during simulated driving: a demonstration. Int J Psychophysiol, 2000, 37, 155-162.

[9] Lin, C.T.; Chung, I.F.; Ko, L.W.; Chen, Y.C.; Liang, S.F.; Duann, J.R. EEG-based Assessment of Driver Cognitive Responses in a Dynamic Virtual-Reality Driving Environment, IEEE Trans Biomed Eng, 2007, 54(7), 1349-1352.

[10] Polich, J.; Kok, A. Cognitive and biological determinants of P300: an integrative review, Biol Psychol, 1995, 41(2), 103-146.

[11] Bouchner, P. A complex analysis of the driver behavior from simulated driving focused on fatigue detection classification, WSEAS Trans on Systems, 2006, 5(1), 84-91.

[12] Egelund, N. Spectral analysis of heart rate variability as an indicator of driver fatigue. Ergonomics, 1982, 25(7), 663-672.

[13] Pomeranz, B.; Macauly, R.J.; Caudill, M.A.; et al. Assessment of autonomic function in humans by heart rate spectral analysis, Am J Physiol Heart Circ Physiol, 1985, 248(1), 151-153.

[14] Kobayashi, H.; Ishibashi, K.; Noguchi, H. Heart Rate Variability; An Index for Monitoring and Analyzing Human Autonomic Activities, J Physiol Anthropol Appl Hum Sci, 1999, 18(2), 53-59.

[15] Michael, G.H. Coles and Michael, D.Rugg. Event-related brain potentials: an introduction, Electrophysiology of Mind. Oxford Scholarship Online Monographs, 1996, 1-27.

[16] Murata, A.; Uetake, A.; Takasawa, Y. Evaluation of mental fatigue using feature parameter extracted from event-related potential, Int J Ind Ergon, 2005, 35(8), 761770.

[17] Engin, A.; Dery, A. A novel approach for digital radio signal classification: Wavelet packet energy-multiclass support vector machine (WPE-MSVM), Expet Syst Appl, 2008, 34, 2140-2147.

[18] Mallat, S. A Wavelet Tour of Signal Processing, 2nd Edition, Academic Press, San Diego, 1999.

() 2010 by NWPII. All rights reserved. 
[19] Shen, M.; Sun, L.; Chan, F.H.Y. Method for extracting time-varying rhythms of electroencephalography via wavelet packet analysis, IEE Proc., Sci. Meas Technol, 2001, 148(1), 23-27.

[20] Rosso, O.A.; Martin, M.T.; Plastino, A. Brain electrical activity analysis using wavelet based informational tools, Physica A : Statistical Mechanics and its Applications, 2002, 313(3), 587-608.

[21] Lal, S.K.L.; Craig, A. Driver fatigue: electroencephalography and psychological assessment, Psychophysiology, 2002, 39(3), 313-321.

[22] Vidyut, G.; Paras, T.; Phalguni, G. Face Classification using Gabor Wavelets and Random Forest, Canadian Conference on Computer and Robot Vision, Kelowna, BC, Canada, 2009, 68-73.

[23] Breiman, L. Random Forests. Machine Learning, 2001, 45(1), 5-32.

[24] Li, Z.Y.; Jiao, K.; Chen, M.; Wang, C. Effect of magnitopuncture on sympathetic and parasympathetic nerve activities in healthy drivers assessment by power spectrum analysis of heart rate variability, Eur J Appl Physiol, 2003, 88(4-5), 404-410.

[25] Craig, A.; Tran, Y.; Wijesuriya, N., Boord, P. A controlled investigation into the psychological determinants of fatigue. Biol Psychol, 2006, 72(1), 78-87.

[26] Oron-Gilad, T.; Ronen, A. Road characteristics and driver fatigue: a simulator study, Traffic Inj Prev, 2007, 8(3), 281-189.

[27] Pilar, T.; Mariano, C. Driving on the motorway: the effect of alternating speed on driver's activation level and mental effort, Ergonomics, 2002, 45(9), 605-618.

[28] Ting, P.H.; Hwang, J.R.; Doong, J.L.; Jeng, M.C. Driver fatigue and highway driving: a simulator study. Physiol Behav, 2008, 94(8), 448-453.

[29] Apparies, R.J.; Riniolo, T.C.; Porges, S.W., A psychophysiological investigation of the effects of driving longer-combination vehicles. Ergonomics, 1998, 41(5), 581-529.
[30] Hartley, L.R.; Arnold, P.K.; Smythe, G.; Hansen, J. Indicators of fatigue in truck drivers. Appl Ergon, 1994, 25(3), 143-156.

[31] Bilchick, K.C.; Berger, R.D. Heart Rate Variability, J Cardiovasc Electgrophysiol, 2006, 17(6), 691-694.

[32] Kleiger, R.E.; Stein, P.K.; Bigger, J.T. Heart Rate Variability: Measurement and Clinical Utility, The Annals of Noninvasive Electrocardiology, 2005, 10(1), 88-101.

[33] Martinmaki, K.; Rusko, H.; Kooistra, L.; Kettunen, J.; Saalasti, S. Intraindividual validation of heart rate variability indexes to measure vagal effects on hearts, Am J Physiol Heart Circ Physiol, 2006, 290(2), 640-647.

[34] Morris, A.M.; So, Y.; Lee, K.A.; Lash, A.A.; Becker CE. The P300 event-related potential The effects of sleep deprivation, J Occup Environ Med, 1992, 34(12), 1143-1152, 1992.

[35] Hsieh, L.; Young, R.A.; Bowyer, S.M.; Moran, J.E.; et al. Conversation effects on neural mechanisms underlying reaction time to visual events while viewing a driving video: An fMRI study, Brain Res, 2009, 1251, 162-175.

[36] Bowyer, S.M.; Hsieh, L.; Moran, J.E.; Young, R.A.; et al. Conversation effects on neural mechanisms underlying reaction time to visual events while viewing a driving scene using MEG, Brain Res, 2009, 1251, 151-161.

[37] Smith, M.E.; Gevins, A. Neurophysiologic monitoring of mental workload and fatigue during operation of a flight simulator. Proceedings of the SPIE, 2005, 5797, 116-126.

[38] Okogbaa, O.G.; Shell, R.L.; Filipusic, D. On the investigation of the neurophysiological correlates ofknowledge worker mental fatigue using the EEG signal, Appl Ergon, 1994, 25(6), 355-365.

[39] Gevins, A.S.; Schaffer, R.E. Critical review of electroencephalographic (EEG) correlates of higher cortical functions, CRC, Cirt Rev Bioeng,1980, 4(2), 113-164.

[40] Vladimir,S.; Andy,L.; Christopher,T.; et al. Random Forest: A Classification and Regression Tool for Compound Classification and QSAR Modeling. J Chem Inform Comput Sci, 2003, 43(6), 1947-1958. 